\title{
Noise Suppression Scheme using Median Filter in Gray and Binary Images
}

\author{
Dr. E. Chandra, \\ Supervising Guide, Director \\ Department of Computer Science, \\ DJ Academy for Managerial Excellence, \\ Coimbatore, Tamilnadu, India
}

\author{
K. Kanagalakshmi \\ Doctoral Research Scholar \\ Department of Computer Science, \\ DJ Academy for Managerial Excellence, \\ Coimbatore, Tamilnadu, India
}

\begin{abstract}
The Quality factor always integrates performance of everything. The performance of a fingerprint image matching process relies on the quality of the fingerprint image. The poor quality image does not define a good structure of the fingerprint image; and the noisy images affect the reliability of Automated Authentication and the Identification systems. To get a good quality image, noises should be suppressed. In this paper, a new scheme is generated to eliminate noise using Median filter. We made an attempt to apply the new scheme on both the gray-scale image and the binary image in order to get better and accurate fingerprint features for further process. The experimental result shows the expected measures such as effectiveness and performance on the median filter using the statistical correlation factor and the computational time among the gray and binary images. The proposed algorithm was implemented using MATLAB 7.10.0 tool. The experimental results show that the algorithm for the enhancement of gray level image gives effectiveness and no loss of information, whereas there is some loss of information in the binary image.
\end{abstract}

\section{General Terms}

Biometrics; Fingerprint Image Enhancement; Noise Removal.

\section{Keywords}

Correlation, Fingerprint, Gray-Image, Binary-Image, Median filter.

\section{INTRODUCTION}

While biometric identification and authentication provide considerable convenience and also some security benefits over token-based or password-based methods, other security and privacy concerns unique to biometrics must also be taken into account. These include identity theft, cross matching, and exposure, often irrevocable, of sensitive private information, as well as trace-ability of individuals. This has stimulated research on the protection of stored biometric data in recent years, primarily focusing on prevention of information leakage. Finger print recognition is a gifted feature for the biometric identification and authentication systems.

The field of biometric is still in its formative years, it is unavoidable that biometric systems will play a significant role in the security [1]. A biometric system is fundamentally pattern recognition system that functions by obtaining biometric data from an individual, extracting a feature set from the obtained data, and evaluating this feature set against the template set in the database [2]. The biometric data comprises fingerprints [3], facial features, iris [4], hand geometry [5], voice [6], and signature [7]. Biometrics is extensively employed in forensics, in criminal identification and prison security to quote a few of the instances, and has the prospect of being employed in a wide variety of civilian application areas. The primary focus of this research article is to provide the finger print classifications, characteristics and to perform the finger print image preprocessing for image enhancement using the median filter. The rest of this paper is organized as follows: Section 2 describes the characteristics of fingerprint. Section 3 shows the literature survey on fingerprint enhancement techniques. Section 4 defines the proposed method using median filter on the finger print image. Section 5 describes the experimental result like the noise rate and the performance of the median filter; Section 6 gives results and discussions and Section 7 concludes this paper. Finally, we include experimental outcomes in the Appendix.

\section{FINGERPRINT}

Fingerprints were accepted formally as valid personal identifier in the early twentieth century and have since then become a defacto authentication technique in law-enforcement agencies worldwide. The FBI currently maintains more than 400 million fingerprint records on file. Fingerprints have several advantages over other biometrics, which are as follows [8]:

- High universality

- High distinctiveness

- High permanence

- $\quad$ Easy collectability

- High performance

- Wide acceptability

At the age of seven months, the fingerprints of a foetus are fully developed. The characteristics of fingerprint do not change throughout the lifetime except for injury, disease, or decomposition after death. However, after a small injury on the fingertip, the pattern will grow back as the fingertip heals [9].

It is supposed that fingerprints are distinct across individuals and across the fingers of a particular individual [10]. It has been established that even identical twins with identical DNA possess different fingerprints. Since many existing fingerprint authentication systems are based on minutiae, which are feature 
points extracted from a raw fingerprint image, a fingerprint can be defined as a pattern of ridges and valleys on the tip of the finger. A fingerprint is, therefore, described by the distinctiveness of the local ridge features and their relationships. Minutiae denote these local ridge characteristics that appear either at a ridge ending or a ridge bifurcation. The point where the ridge comes to an abrupt end is known as ridge ending and the ridge bifurcation is denoted as the point where the ridge divides into two or more branches. Fingerprint usage can be divided into three different areas:

- Security as identification of individuals

- Forensics, also as an identification method

- Personal characteristics often involved with horoscopes and similar non-scientifically proven prophecies.

The first two are by far the greatest areas. Fingerprint-based systems, used for security reasons, are so popular today that they have almost become the synonym for biometric systems.

\subsection{Fingerprint characteristics}

You have probably looked at your own fingerprint at some point in your life and noticed the papillary lines on it. In fingerprint literature, the terms ridges and valleys are used to describe the higher and lower parts of the papillary lines. The reason we have ridges and valleys on our finger, is the frictional ability of the skin. The formation of the ridges and valleys is a combination of genetic and environmental factors. The DNA gives directions in the formation of the skin of the foetus, but the exact formation of the fingerprint is a consequence of random events [10]. This is also the reason why the fingerprints on different finger on the same individual are different, and why identical twins have different fingerprints

\section{RELATED WORK}

Fingerprint image enhancement is a vital process in the biometric systems. Weickert[11] introduced a diffusion technique. It is based on multi-scale analysis which is also called scale-space representation; and also this technique includes an iterative process of local feature estimation. S.Greenberg et al. [12] developed a technique which is based on anisotropic filtering technique; but it does not use diffusion technique for enhancement. Munteanu C. Rosa [13] designed an Evolutionary algorithm for gray-scale enhancement. In this algorithm evolutionary optimization processes were followed. Farbiz et al. [14] proposed a method based on fuzzy-logic-control which is used to eliminate impulsive noise and smooth Gaussian noise and also preserve edges and image details. Jing $\mathrm{Li}$ et al. [15] implemented color based grayscale-fused image enhancement algorithm to stick out the meaningful information of a certain sensor with the high resolution of the grayscale-fused image.

\section{PROPOSED METHOD}

Image preprocessing is an essential part in the Automatic Fingerprint Authentication and Identification systems. Obviously, it needs quality and purity of images. To achieve these factors, fingerprint enhancement is obligatory due to deficiencies of images. Fingerprint Enhancement can be done on either
- Binary ridge Images or

- Gray-level Images.

In a gray-level fingerprint image, the ridges and valleys in a local neighborhood form a sinusoidal-shaped plane wave which has a well-defined frequency and orientation.. In this paper, we propose a new method and analyze the performance of the method on gray and binary images through the experiments. The goal of the enhancement algorithm is to improve the accuracy and suppress the undesired noisy portions of all structures of finger print images in the desired recoverable regions. So that the quality of the features such as ridges, valleys and extended features- pores and contours- are important for further matching process. The objective of our proposed method is to provide a noise-free good quality image. Our proposed scheme includes the steps as specified in the algorithm.

\subsection{Proposed Algorithm}

In this section the proposed algorithm describes the process of suppressing noises in gray-level images; and also suppression of noises in the Binary images. The flowchart of the proposed algorithm is shown in figure 1 .

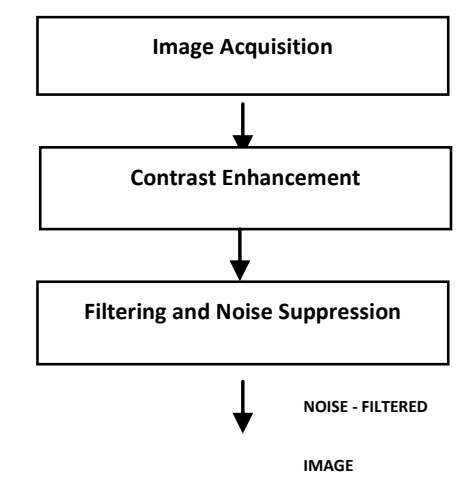

Figure 1: Flowchart for Algorithm

The main steps of the algorithm include:

1. Image Acquisition.

2. Contrast Enhancement (Histogram equalization).

3. Apply the Median Filtering on gray-scale image and suppress noises.

4. Get a Median Filtered quality Gray-scale image.

- Image Acquisition: Images are obtained by using either live fingerprint scanner or from the database which contains collection of fingerprint images. For our experiment, we obtained fingerprint images from the livefingerprint reader and also used ${ }^{1} \mathrm{FVC} 2002$ DB1 fingerprint images.

\footnotetext{
${ }^{1}$ Fingerprint Verification Contest 2002 Database 1
} 
- Contrast Enhancement (Histogram equalization) on the gray-level image: Contrast enhancement is carried out through histogram equalization on the gray image. Histogram equalization is the process of distributing the levels of brightness for the pixels in order to increase the brightness of the image.

- $\quad$ Filtering and Noise Removal (Median Filtering): After completion of histogram equalization on image, Median filter is applied to the image to eliminate noises. Median Filter is a non-linear smoothing method that reduces the blurring of edges, in which the idea is to replace the current point in the image by the median of the brightness in its neighborhood. Individual noise spikes do not affect the median of the brightness in the neighborhood and so median smoothing eliminates impulse noise quite well [16]. Median filter is a better filtering technique according to performance and takes less computational time. It smooths salt and pepper noises [17]. In our experiment, the median filter was applied and we got the median filtered fingerprint image as a good quality image for further enhancement based on the expression 1.During the process of median filtering, each pixel is replaced by the median of the pixels contained in a window around it. It can be expressed as:

$\operatorname{MM}(m n)=\operatorname{Median}\left[x\left(m-\mathbb{k}_{w} n-\mathbb{P}\right) \in W\right]$

Where $\mathrm{w}$ is the window around the pixels $\mathrm{m}, \mathrm{n}$.

- $\quad$ Filtered cum Quality Image: After filtering, a good and noise suppressed image can be obtained without affecting the information of ridges.

\section{EXPERIMENTAL TASKS AND RESULTS}

This section describes the experimental steps and results obtained. Initially we obtained images from the FVC 2002 Database1. In our experiment, the proposed algorithm is experimented on both the gray level and the binarized images using MATLAB 7.10.0 Tool.

Experiment 1 on Gray-level images: The developed algorithm is applied on gray level images. Initially we captured live fingerprint images using fingerprint reader and also obtained several images from the FVC 2002 Database1. And we observed the quality cum noise free images on the gray-level with no loss of information on fingerprint.

Experiment 2 on Binary Images: The algorithm is also implemented in the binary images. During this attempt, steps 1 and 2 of the algorithm are followed as they are. After that the histogram equalized gray-level image [range $0-255$ ] is converted into the binary image (called Binarization). Binarization is the process of transforming the Gray-scale image into the binary image $[0,1]$. New value $(0$ or 1$)$ can be assigned for each pixel according to the intensity mean in a local neighborhood, as follows:

$I_{\text {MeV }}\left(p 1_{s} p 2\right)=\left\{\begin{array}{l}1 \text { if } I_{\text {old }\{1} 1 \text { p2ziocal Mean } \\ 0 \text { otherwise }\end{array}\right.$

The gray-scale transformations do not depend on the position of the pixel in the image. During the binarization process, the low frequency pixels may be omitted. Those pixels may be right feature or an important feature for future process [see figure 3, 4 and 5 of APPENDIX]. Performance of the median filtering and the algorithm process efficiency are measured in terms of the statistical-correlation, computational factor and pixel values cross-section along the line segments between gray and binary images.

\section{- PERFORMANCE MEASURE 1: Correlation Factor}

The correlation value for each fingerprint images (After Histogram Equalization) which is obtained in our experiment is shown in the table 1 . The table values also show the algorithm's efficiency on gray-level image and the binary images. Result shows the changes made in the original image while implementing the algorithm (using Median filter). The performance analysis of median filter for ten fingerprint images is shown in figure 2 . The correlation factor is calculated using the following procedure.

$R=\frac{\sum_{0 \leq i \leq m}\left(A_{m m}-A v\right)\left(B_{\mathrm{mm}}-B v\right)}{\sqrt{\sum_{0 \leq i \leq \mathrm{m}}\left(A_{\mathrm{mm}}-A v\right)^{2}\left(B_{\mathrm{mm}}-B v\right)^{2}}}$

Where $\mathrm{A}=$ Original (Noisy) Image, $\mathrm{B}=$ Filtered Image, $\mathrm{m}, \mathrm{n}=$ size of the Images; $A^{`}=$ Mean $(A)$ and $B^{`}=$ Mean $(B)$. Using the equation (3), correlation coefficient between Gray level image and Binary image (the same image processed with binarization). Both the images were processed under the algorithm. 
Table 1 Correlation between Original Noisy Image and the Median Filtered Image

(For both Gray-Level and Binary Images)

\begin{tabular}{ccc}
\hline $\begin{array}{c}\text { Fingerprint } \\
\text { Image \# }\end{array}$ & $\begin{array}{c}\text { Noisy- Gray Image and } \\
\text { Median Filtered Image }\end{array}$ & $\begin{array}{c}\text { Noisy- Binary } \\
\text { Image } \\
\text { and Median } \\
\text { Filtered Image }\end{array}$ \\
\hline $101 \_1$ & 0.9639 \\
$102 \_1$ & 0.9802 & 0.9756 \\
$103 \_1$ & 0.9881 & 0.9802 \\
$104 \_1$ & 0.9934 & 0.9675 \\
$105 \_1$ & 0.9876 & 0.9656 \\
$106 \_1$ & 0.9857 & 0.9735 \\
$107 \_1$ & 0.9918 & 0.9597 \\
$108 \_1$ & 0.9815 & 0.9696 \\
$109 \_1$ & 0.9875 & 0.9546 \\
$110 \_1$ & 0.9682 & 0.9535 \\
\hline
\end{tabular}

Correlation fator between gray, Binary with their Median Filtered Images

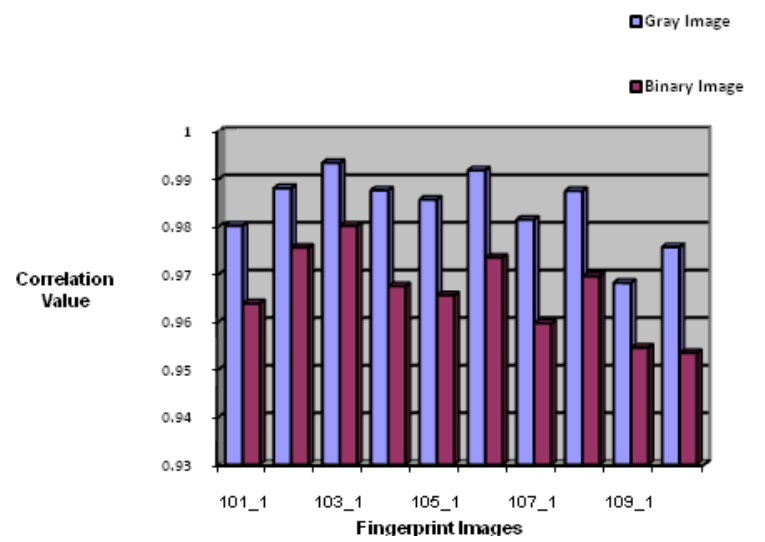

Figure 2 Performance Analysis chart for Gray-level and Binary Image (After Histogram Equalization)

- PERFORMANCE MEASURE 2: Computational time The performance of the Median filter on the gray-level and the binary image is identified using the correlation factor and computational time. The computational time of the Median filter is listed in the table 2. According to computational time, the median filter takes the same amount of seconds for both the gray-level and binary images. But in terms of correlation factor, gray-level is higher than the binarized images.
Table 2 Computational time of Median Filter on ten FVC2002 DB fingerprint images with 0.00000004 s clock precision and $2805 \mathrm{MHz}$ clock speed (in Pentium(R)) for both Gray and Binarized Images

\begin{tabular}{ccc}
\hline $\begin{array}{c}\text { Fingerprint } \\
\text { Image \# }\end{array}$ & $\begin{array}{c}\text { Total } \\
\text { Recorded } \\
\text { Time }\end{array}$ & $\begin{array}{c}\text { Computational Time } \\
\text { for Noise Removal }\end{array}$ \\
\hline $101 \_1$ & $0.1 .64 \mathrm{~s}$ & $0.04600000 \mathrm{~s}$ \\
$102 \_1$ & $0.1 .69 \mathrm{~s}$ & $0.04600000 \mathrm{~s}$ \\
$103 \_1$ & $0.1 .69 \mathrm{~s}$ & $0.04600000 \mathrm{~s}$ \\
$104 \_1$ & $0.1 .70 \mathrm{~s}$ & $0.04600000 \mathrm{~s}$ \\
$105 \_1$ & $0.1 .95 \mathrm{~s}$ & $0.04700000 \mathrm{~s}$ \\
$106 \_1$ & $0.1 .78 \mathrm{~s}$ & $0.04700000 \mathrm{~s}$ \\
$107 \_1$ & $0.1 .70 \mathrm{~s}$ & $0.04600000 \mathrm{~s}$ \\
$108 \_1$ & $0.1 .67 \mathrm{~s}$ & $0.04700000 \mathrm{~s}$ \\
$109 \_1$ & $0.1 .61 \mathrm{~s}$ & $0.04700000 \mathrm{~s}$ \\
$110 \_1$ & $0.1 .69 \mathrm{~s}$ & $0.04700000 \mathrm{~s}$ \\
\hline
\end{tabular}

- PERFORMANCE MEASURE 3: Computing Pixelvalue cross-sections

For further analysis on the Noise suppression on gray-level and the binary images, we computed pixel-value cross-sections along line segments. The xi and yi are (equal-length) vectors specifying the spatial coordinates of the endpoints of the line segments; and computed the intensity values along a line or a multiline path in a gray-level, Median Filtered Gray-level image, Bianry image, and Median filtered Binary Image (see figure 3,4 and 5 in APPENDIX).

\section{RESULTS AND DISCUSSIONS}

In summary, the algorithm is implemented in both the graylevel and the binary images. From the experimental results, it is verified that the enhancement process on the gray-level image can provide better cum lossless sensitive features of the image than the binary image. Because of the conversion taken place in the binary image, the image may lose its important features or information. The experiment pixel value cross-section (see fig. 3,4 , and 5) clearly shows the omitted pixels in the binary image but not in the gray-level image during the enhancement. Those pixels may be true pixels which were eliminated during the binarization and enhancement of the same. Thus, it is validated that the enhancement in gray-level image is most preferable. But after the enhancement, the gray-level image can be converted into binary image for other image processing in order to get sharp, thinned patterns and reduced size.

\section{CONCLUSION}

The experimental result shows that applying enhancement and the filtering techniques on the gray-level images can provide a better noise suppression with restoration of original information of the ridge patterns of the fingerprint images than binary images. Based on experimental results the algorithm can provide a better performance. 


\section{ACKNOWLEDGEMENT}

The Author acknowledges her thanks to her guide Dr.E.Chandra for her enlightened guidance. She would like to thank her Secretary, Principal, all faculty members and friends for their valuable support. Author uniquely likes to thank Prof.S.Chinthamani, Principal and HOD of English (VCAS) who assist her to produce a flawless content. Finally she extends her gratitude to the members of her family for their excellent moral support.

\section{REFERENCES}

[1] John Chirillo and Scott Blaul, Implementing Biometric Security, Wiley Red Books, (2003), ISBN: 9780764525025 .

[2] Jain, A.K., Ross, A. and Prabhakar, S, An introduction to biometric recognition, IEEE Transactions on Circuits and Systems for Video Technology, (2004), Vol. 14, No. 1, pp: 4- 20.

[3] T. C. Clancy, N. Kiyavash, and D. J. Lin, Secure smartcardbased fingerprint authentication, in Proceedings of the ACM SIGMM Workshop on Multimedia Biometrics Methods and Applications (WBMA '03), (2003), pp. 4552 .

[4] A. Goh, D.C.L. Ngo, Computation of cryptographic keys from face biometrics, International Federation for Information Processing, Springer-Verlag, LNCS 2828, (2003), pp. 1-13.

[5] Övünç Polat and Tülay Yýldýrým, Hand geometry identification without feature extraction by general regression neural network, Expert Systems with Applications,(2008), Vol. 34, No. 2, pp. 845-849.

[6] F. Monrose, M.K. Reiter, Q. Li and S. Wetzel, Cryptographic key generation from voice, Proceedings of the 2001 IEEE Symposium on Security and Privacy, (2001), pp.202-213.

[7] S. Pankanti, S. Prabhakar, A.K. Jain, On the individuality of fingerprints, IEEE Trans. Pattern Analysis and Machine Intelligence, (2002),vol. 24, no. 8, pp.1010-1025.

[8] Tsai-Yang Jea, Minutiae Based Partial Fingerprint Recognition, Thesis.
[9] Sharat S. Chikkerur, Online Fingerprint Verification System, Thesis.

[10] Raju Sonavane, B.S.Sawant, Noisy Fingerprint Image Enhancement Technique for Image Analysis: A Structural Similarity Measure Approach, International Journal of Computer Science and Network Security, (2007), Vol.7, No.9, pp.225 - 230.

[11] Weickert, J., Applications of nonlinear diffusion in image processing and computer vision, ACTA Mathematica Universitatis Comenianae, (2001), Vol. 70, Issue 1, pp: 3350.

[12] S.Shlomo Greenberg, Mayer Aladjem and Daniel Kogan, Fingerprint Image enhancement using filtering Techniques, Journal of Real Time Imaging, Elsevier, (2002), pp.227236.

[13] Munteanu, C. Rosa, A., Gray-scale image enhancement as an automatic process driven by evolution, IEEE Transactions on Systems, Man, and Cybernetics, (2004) Vol. 34, Issue 2, pp.1292 -1298.

[14] Farbiz, F. Menhaj, M.B. Motamedi, S.A. Hagan, M.T., IEEE Transactions on Systems, Man, and Cybernetics,(2002), Vol.30, issue 1, pp.110-119.

[15] Jing Li, Quan Pan, Tao Yang, Yong mei Cheng, Color based grayscale-fused image enhancement algorithm for video surveillance,(2004),Proceedings of Third International Conference on Image and Graphics, pp.47 50, doi:10.1109/ICIG.2004.45.

[16] E.Chandra and K.Kanagalakshmi, Noise Elimination in Fingerprint Images using Median Filter, Int. Journal of Advanced Networking and Applications,(2011),Vol. 02, Issue:06, pp:950-955.

[17] E.Chandra and K.Kanagalakshmi, Performance Evaluation of Filters in Noise Removal of Fingerprint Image, Proceedings of ICECT-2011, $3^{\text {rd }}$ International Conference on Electronics and Computer Technology,(2011), vol. 1, pp. 117-123, ISBN: 978-1-4244-8677-9, Published by IEEE, Catalog no.: CFP1195F-PRT. 


\section{APPENDIX}

Exprimental results are observed and sample results are shown below (see figure 3,4, and 5) for the human perception.

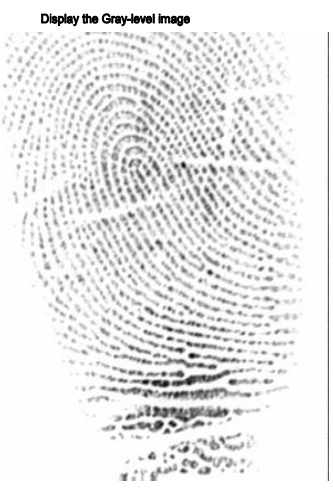

(a)

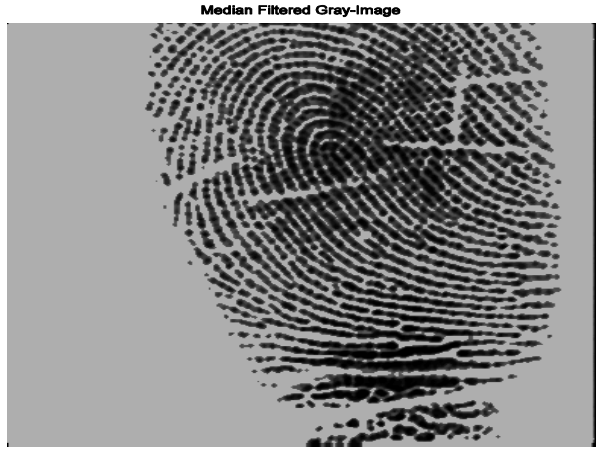

(c)

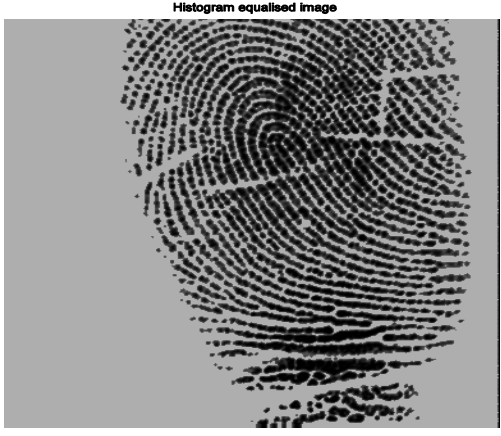

(b)

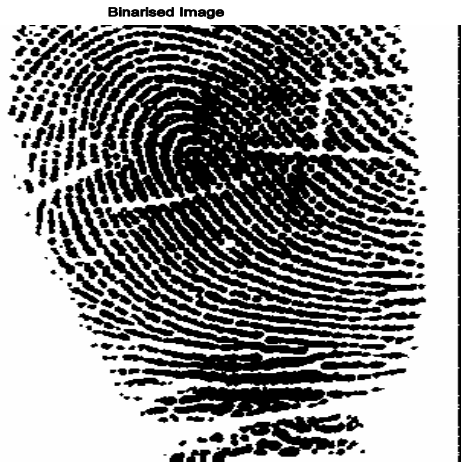

(d)

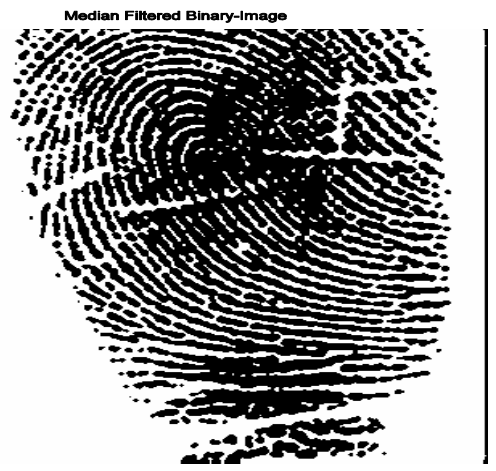

(e)

Figure 3 shows the pixel representation in gray-level and binarized image and median filtered image:

(a) Original Image (FVC 2002 DB1:110_1.tiff), (b) Histogram Equalized Image (c) Median Filtered Gray-Level Image (d) Binarized Image (e) Median Filtered Binary Image. 


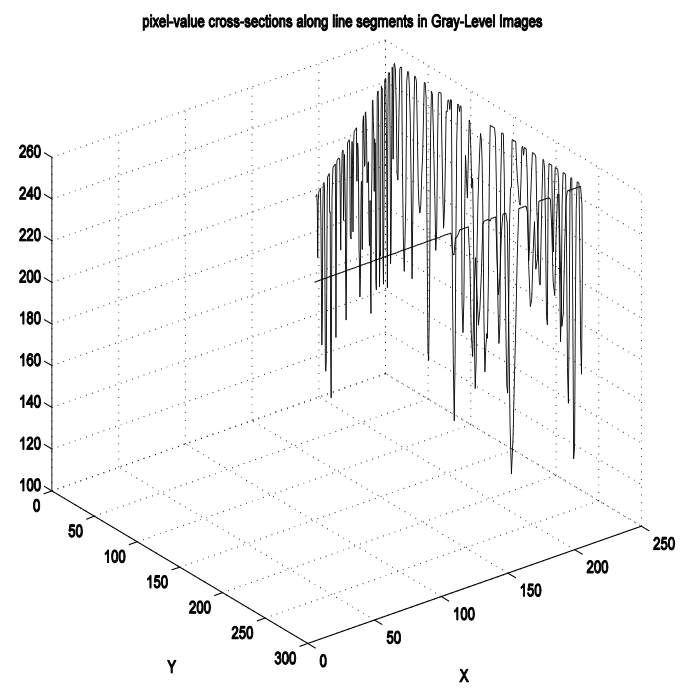

(a)

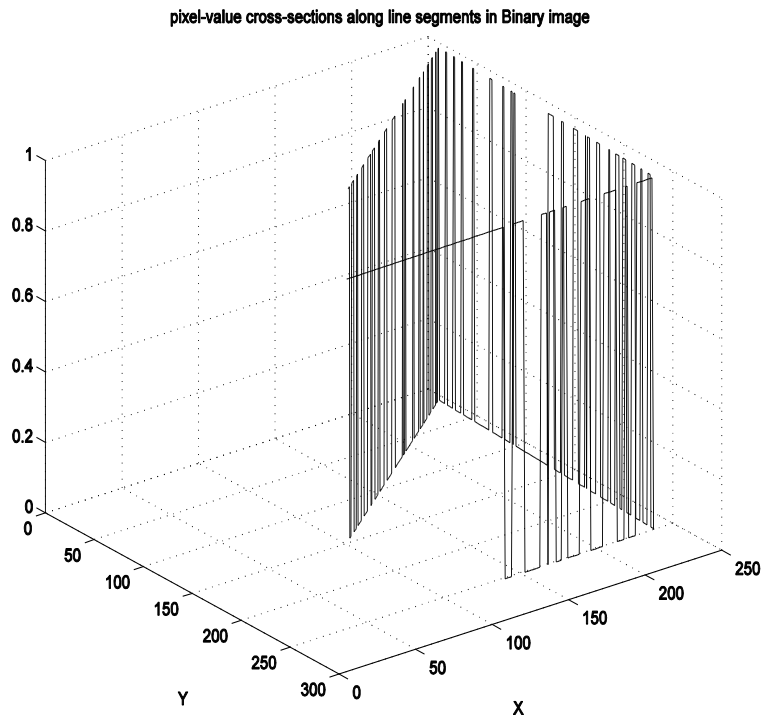

(c)

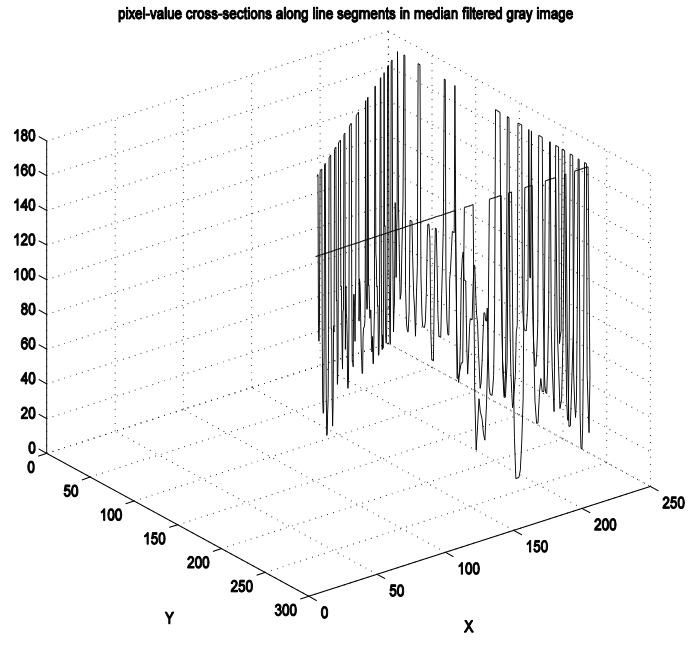

(b)

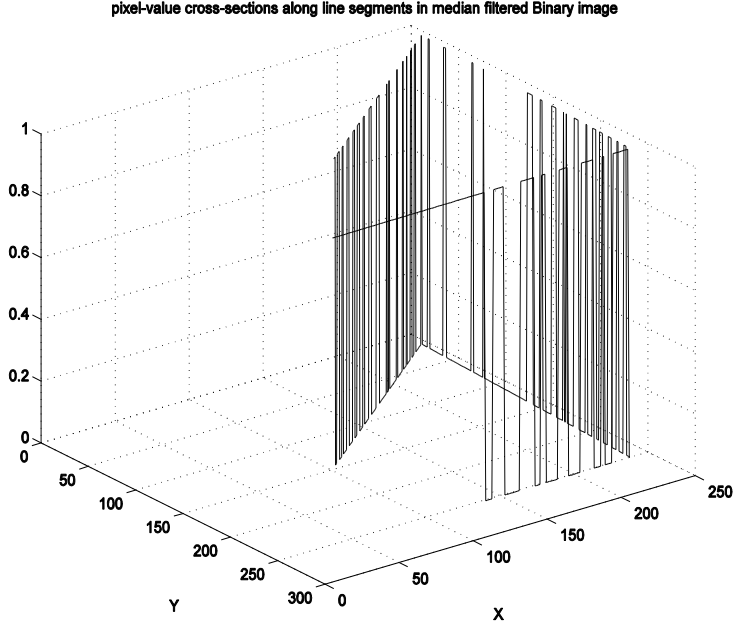

(d)

Figure 4 shows the pixel value cross-sections along line segments xi = [35 238246 103]; yi = [253 25017 148] :

(a) Gray-Level Image [FVC DB1: 110_1.tiff] (b) Median Filtered Gray-level Image (c) Binary Image (d) Median Filtered Binary Image 


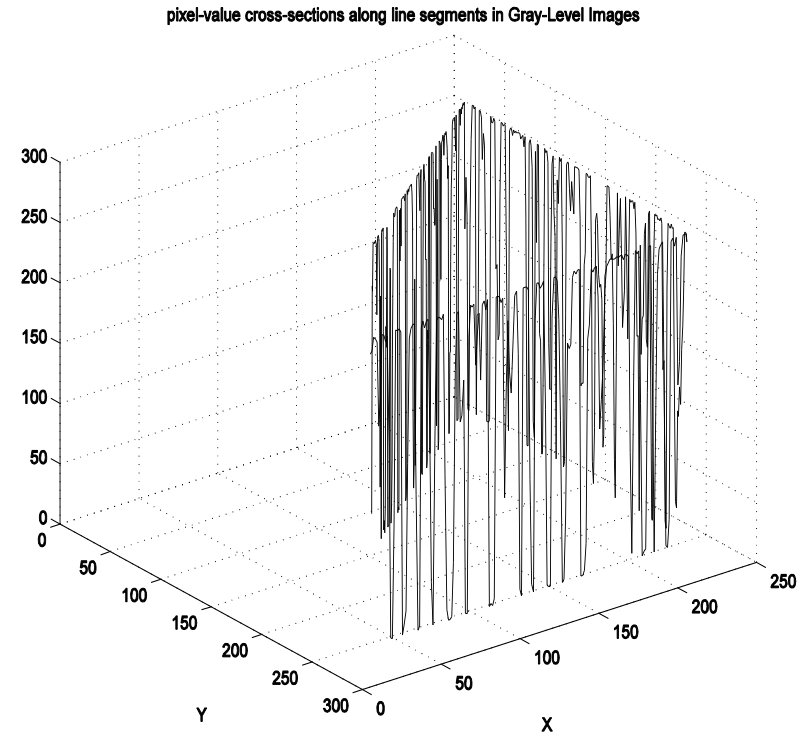

(a)

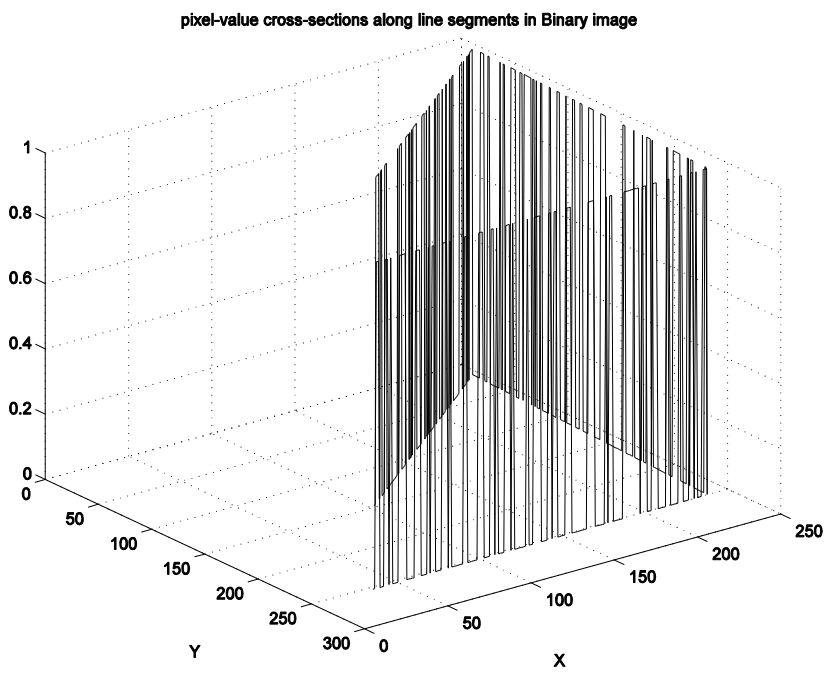

(c)

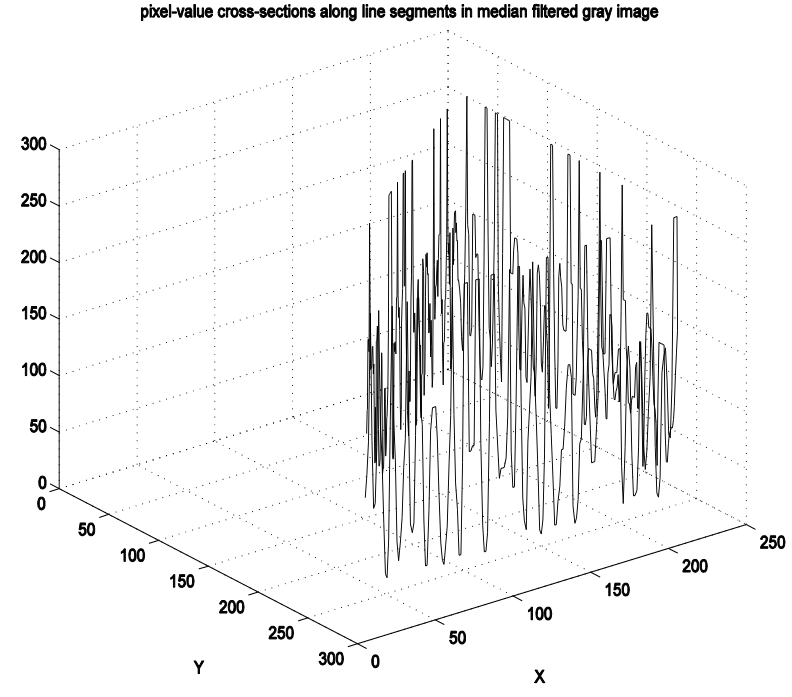

(b)

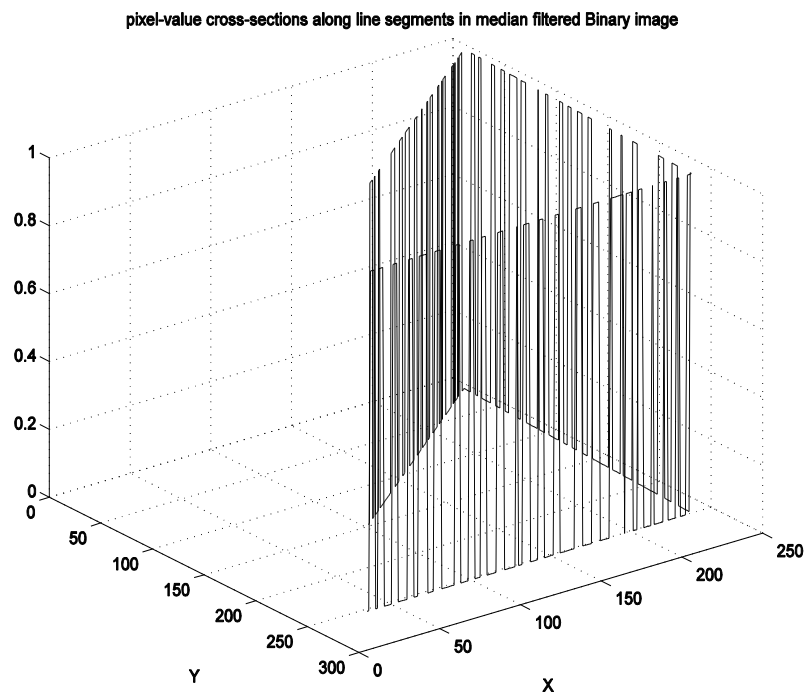

(d)

Figure 5 shows the pixel value cross-sections along line segments xi = [35 238246 103]; yi = [253 25017 148]:

(a) Gray-Level Image (Real fingerprint captured from fingerprint reader:fp1.tiff) (b) Median Filtered Gray-level Image (c) Binary Image (d) Median Filtered Binary Image 


\section{AUTHOR PROFILE:}

Dr.E.Chandra received her B.Sc., from Bharathiar University, Coimbatore in 1992 and received M.Sc., from Avinashilingam University, Coimbatore in 1994. She obtained her M.Phil, in the area of Neural Networks from Bharathiar University, in 1999. She obtained her PhD degree in the area of Speech recognition system from Alagappa University Karikudi in 2007. At present she is working as a Director in the Department of Computer Science at D. J. Academy for Managerial Excellence, Coimbatore. She has published more than 30 research papers in National, International journals and conferences. She has guided more than 30 M.Phil, research scholars. At present, she is guiding $8 \mathrm{PhD}$ research scholars. Her research interest lies in the area of Data Mining, Artificial intelligence, neural networks, speech recognition systems and fuzzy logic. She is an active member of CSI, Currently management committee member of CSI, Life member of Society of Statistics and Computer Applications.

Ms.K.Kanagalakshmi, completed her B.Sc. in Madurai Kamaraj University, Madurai, and MCA degree in Bharathiar University, Coimbatore, and M.Phil Degree in Madurai Kamaraj University, Madurai. She is working as an Assistant Professor in the Department of Computer Science, Vidyasagar College of Arts and Science, Udumalpet, Thirupur (DT), Tamilnadu. She is a Doctoral research scholar of DJ Academy, Coimbatore. She has produced two M.Phil Scholars. She has presented 30 papers in National and International conferences and Journal. Her Area of research is Biometrics and security. Other areas of interest are Computer and Information Security, Image Processing. She is an Associate Member of CSI. 